\title{
Experimental analysis of the Portevin Le Chatelier effect by means of full field non contact techniques
}

\author{
F. Curà ${ }^{1}$, P. Matteis ${ }^{2}$, G. Scavino ${ }^{2}$, R. Sesana ${ }^{1}$ \\ ${ }^{1}$ Politecnico di Torino, Dip. di Meccanica1, C.so Duca degli Abruzzi 24, 10129 Torino, Italy \\ ${ }^{2}$ Politecnico di Torino, Dip. di Scienza dei Materiali e Ingegneria Chimica2, C.so Duca degli \\ Abruzzi 24, 10129 Torino, Italy
}

\begin{abstract}
Strain related phenomena in materials showing the Portevin Le Chatelier (PLC) effect are investigated when subjected to loading conditions both in elastic and in plastic field. Two experimental techniques for strain measurements are compared, thermography and optical strain measurement, both non contact, non destructive and full field. Two materials have been tensile tested with several test speeds. Each specimen has been observed and test evolution has contemporaneously been acquired with both video and thermotracer. Each test has been repeated several times. Aim of this research is to investigate how these experimental techniques may quantitatively describe the above described phenomena. Experimental data are useful to calibrate models describing the mechanical behaviour of materials (i.e. constitutive laws, damage models, etc.) and to focus phenomena which take place during load and corresponding strain phases.
\end{abstract}

\section{Introduction}

Strain values measurements in specimens and components, when subjected to loading conditions both in elastic and in plastic field, are an important source of information. These experimental data are useful to calibrate models describing the mechanical behaviour of materials (i.e. constitutive laws, damage models, etc.) and to focus phenomena which take place during load and corresponding strain phases. When restrictive aesthetic or processing requirements are prescribed, as in steels pressing and drawing, particular interest has to be devoted to related physical phenomena.

TWIP austenitic steels (containing Fe e Mn [1]) have been concept and developed for automotive industries, showing an higher tensile resistance with respect to common steels, a very good elongation at break and a good ductility during moulding.

Exceptional mechanical properties of TWIP steels may be given for as concerns microscopic phenomena to two deformation modes of the structure as mechanical gemination and dislocation motion when planar sliding occurs [2, 3].

TWIP steels show also a negative sensitivity to the strain velocity (negative strain rate sensitivity), that is a stress decreasing (for equal strain) for an increasing strain velocity.

Another characteristic of TWIP steels is that may point out the Portevin-Le Chatelier (PLC) effect [4] for particular ranges of strain velocity, loading conditions and temperature. The PLC effect is a plastic instability phenomenon and it is emphasized by a non uniform specimen strain as an unstable plastic flux. The specimen stress may be localized in bands both stationary or propagating along the direction of the tension axis.

This is an Open Access article distributed under the terms of the Creative Commons Attribution-Noncommercial License 3.0, which permits unrestricted use, distribution, and reproduction in any noncommercial medium, provided the original work is properly cited. 
Different kinds of instability may be related to the PLC effect concerning both distribution and evolution of bands along the specimen: "A" bands propagate continuously (solitary plastic waves); "B" bands show an oscillatory or intermitting propagation along the tensile axis (stop-and-go); "C" bands come out with random distribution without any propagation on the specimen (statistical nucleation).

These phenomena are also related to an aging process, known as Dynamic Strain Aging (DSA), that contributes to an increasing of the tensile resistance, to the hardening and to an elongation decreasing in the plastic phase.

The strain behaviour of steels in plastic range has been widely experimentally investigated. However, phenomena related to the PLC effect are not so easily analysed by means of traditional techniques. From the micro structural point of view, are useful microscopy and $\mathrm{X}$ rays diffraction.

A typical problem arising in strain measurements is the influence of the experimental device on the measurement uncertainty. This particularly occurs in the case of contact measurements devices as LVDT, strain gages, etc. Non contact, non destructive and full field experimental techniques as thermography and optical strain measurements may be more satisfactory employed in order to both qualitatively and quantitative measure the above described phenomena [5].

Thermography has already been used to investigate plasticity propagation $[5,6]$ by means of the surface thermal variation during loading conditions. By means of the acquisition of thermal contours sequences it is possible to follow the evolution of the PLC effect.

As an example, in [6, 7] thermography has been utilized to investigate the Lüders bands propagation and a relationship has been found between thermal dissipation and strain velocity.

In [8] a model about the propagation velocity of PLC bands as a function of the test velocity and the number of propagated bands has been proposed. Thermal data acquired during an uniaxial tensile test have been utilized to obtain characteristic parameters of the phenomenon.

The first aim of this paper is to compare the already quoted experimental techniques for strain measurements, thermography and optical strain measurement technique, both non contact, non destructive and full field. These techniques have been applied to the analysis of two materials undergoing tensile tests and showing the PLC effect. The second aim of this research is to investigate how these two techniques can quantitatively describe the phenomena, as an example by means of speed of the plasticity propagation bands, their frequency related to test parameters, etc.

A TWIP steel with and without galvanization treatment has been analysed. Specimens have been tensile tested with several test speeds. Each specimen has been observed and the test evolution has been acquired contemporaneously with both video and thermocamera. Each test has been repeated three times.

\section{Materials and methods}

In the present paper the behaviour of a TWIP steel with and without galvanizing treatment has been investigated. This TWIP steel has been previously tested by means of $\mathrm{X}$ ray diffraction, optical and SEM microscopy to determine grains dimensions and to investigate if grain boundary effects may appear. Then, specimens have been tensile tested with three velocities (respectively $0.05,0.5$ and $5 \mathrm{~mm} / \mathrm{s}$ ) of the crosshead of the testing machine (Wolpert, load cell $600 \mathrm{kN}$ ) in order to evaluate how this velocity may influence the nucleation and propagation of PLC bands and how the employed experimental techniques are useful to point out this phenomenon. For each testing configuration, three specimens have been tested.

To obtain the strain range measurements the strain measurement optical system Aramis (GOM, Optical Measuring Techniques) and a thermotracer NEC Micron 7100V TN7102MX/WX.

Plate specimens have been obtained according to international Standards [9]. Specimens have been prepared for optical strain measurement and for thermographic analysis [5]; in particular they have been polished so as to remove superficial oxide and then they have been painted: one side, that for optical strain measurement, by means of a white spray coating very elastic in order to follow large strains during test and then by means of an uniform film of a black graphite; the opposite side, 
that for thermographic acquisitions, by means of a black paint in order to obtain a superficial emission coefficient equal to 0.9 or higher. Both faces of plate specimens have been monitored by means respectively of the Aramis system and the thermotracer.

Figure 1 shows the above described testing set up.

The PLC effect shows a temperature increase together with the band propagation and the corresponding plastic strain zone, then temperature decreases when strain ends. In figure 2 a thermal image, acquired during a tensile test on a TWIP specimen, is presented as an example. The termal increment corresponding to an "A" PLC band is visible. The direction of propagation is from bottom to top, higher temperature pixel are red and lower yellow to green.

To process thermal data, three reference points along the specimen axis (ROI1, 2, 3), located at a regular distance from the fixed crosshead of the testing machine, have been chosen to identify the temperature increment related to the band propagation and to calculate the corresponding velocity of each band "A". Experimental hypothesis about thermal sources and heat propagation defined in [6, 7] have been taken into account. A band crossing a reference point is identified as a thermal peak in the time-temperature acquisition of the reference point as shown in figure 3. Band propagation velocity is calculated as the ratio between the distance between reference points and the time span in which a band crosses the two reference points. It may be noted that this measure of the band propagation velocity is affected by the velocity of the moving crosshead of the testing machine. The temperature increment related to each band has been recorded. The thermal increment has been calculated between the maximum temperature in a reference point, when the band crosses it, and a point on the specimen which has the minimum temperature. This point is set close to the testing machine grips and its temperature is almost constant during the test.

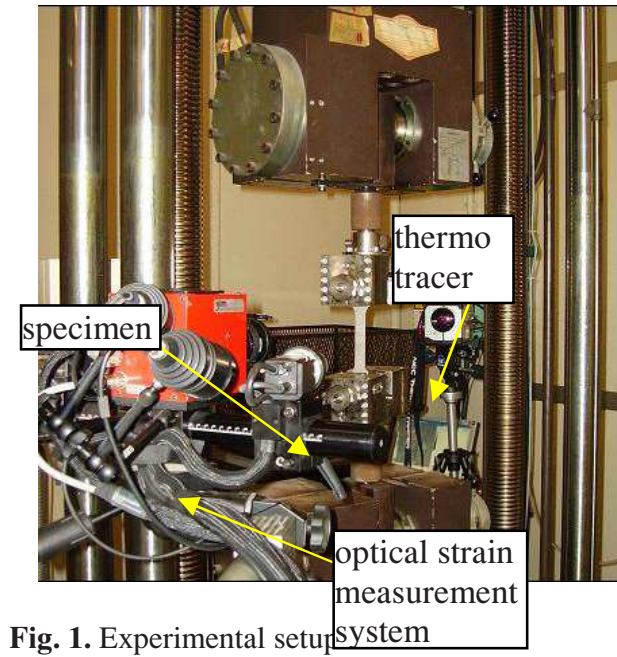

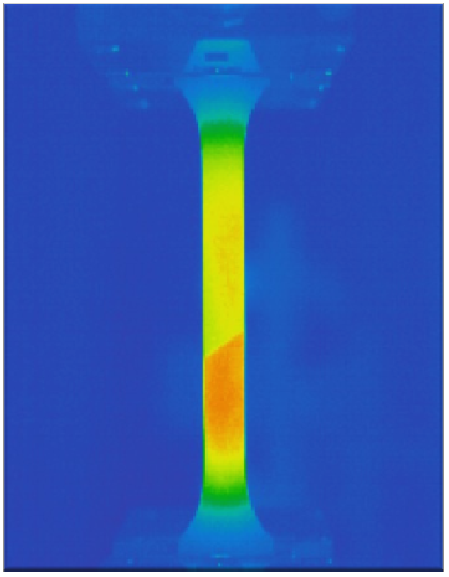

Fig.2. Example of thermal acquisition during "A" band propagation

Optical strain measurements allow to quantify the strain distribution during the test. For sake of time calculation brevity, three parallel equidistant lines with respect to the axis of the specimen have been considered for measurements. The three lines are constituted by a finite number of equidistant points, which distance may be defined by the user. PLC bands propagating in the material cause plastic deformations: the centre of the band in propagating phase corresponds to a maximum of the localized strain. This peak may be identified by calculating the distance between two subsequent points changing in time and by identifying the maximum value along a segment in time. Both strain entity and absolute velocity of the band may be then obtained; it may be pointed out that this measure is not influenced by the velocity of the moving crosshead of the testing machine.

The force-crosshead displacement curve obtained from the testing machine allows to discriminate between "A" and "C" PLC bands. "A" bands are well defined and separated; they start 
to nucleate when plastic strain starts in the specimen. Just before rupture, "C" bands may be recognized as very narrow load peaks as shown in Figure 3.

Four data acquisition (temperature, strain, crosshead displacement and load) have been sincronized. Band propagation velocity measurements obtained by means of thermography and optical strain measurements have been compared along with force measurements corresponding to band propagation speed velocities and temperatures to obtain a detailed description of the experimental aspects of the PLC effect.

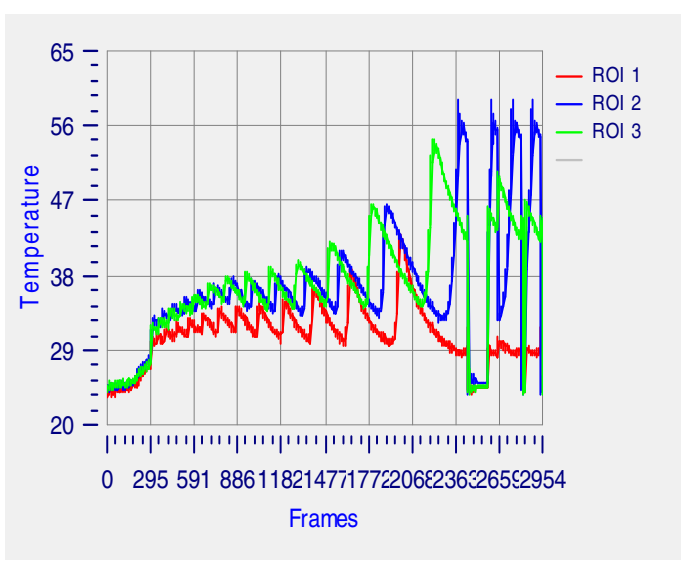

Fig. 3. Temperature acquisition of reference points during tensile test

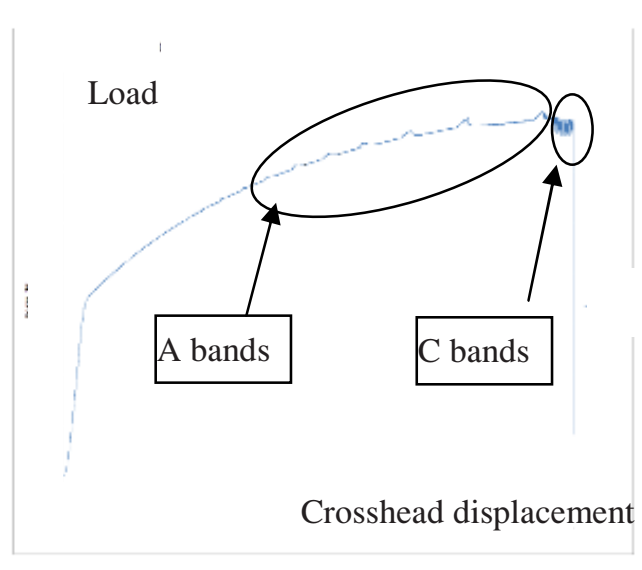

Fig. 4. Load displacement acquisition during tensile test

\section{Results and discussion}

\subsection{Preliminary investigations}

Preliminar diffractometric analysis has shown a full austenitic structure without carbides.

Both optical and SEM microscopy analises has shown that grain dimensions range between 3 and $8 \mathrm{~m}$. The austenitic structure and the absence of carbides have been confirmed.

\section{2 "A" band propagation velocity}

In Figure 5 optical strain measurement results related to a specimen tested with $0.05 \mathrm{~mm} / \mathrm{s}$ test speed are reported as an example. Strain deformation are related to a line parallel to the axis of the specimen in different time instants. Bands propagate from right to left. 
Strain

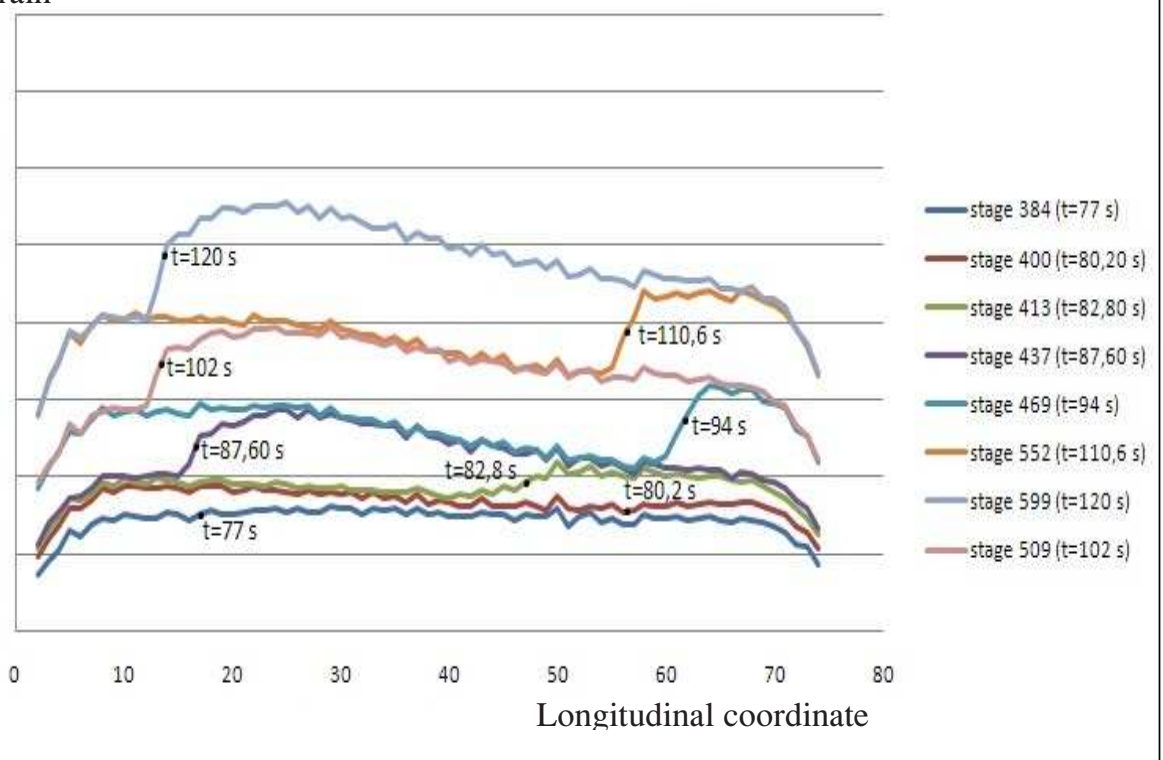

Fig. 5. Optical strain measurements results

In Figure 6, the "A" band propagation velocities obtained by means of the optical strain measurement system are plotted. Each point represents the velocity of a band. Bands related to the same specimen are represented by means of the same symbol. Points related to the same tensile test speed are gathered in a circle.

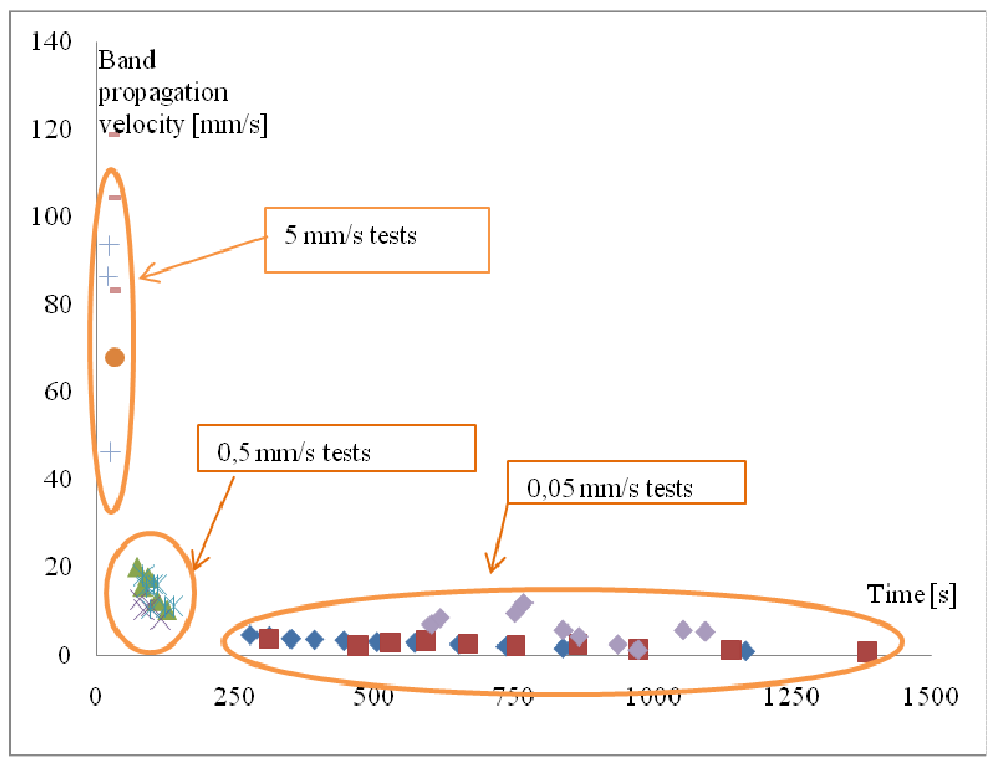

Fig. 6. "A" band propagation speed by means of optical strain measurements. (galvanized specimens. , $\times, \odot$ ) 
PLC "A" bands related the non galvanized specimens nucleates in one end of the specimens and propagate towards the other end. For lower test speed, the "A" bands are numerous and propagate with lower velocities. For example for $0.05 \mathrm{~mm} / \mathrm{s}$ test speed, band propagation velocity ranges about $5 \mathrm{~mm} / \mathrm{s}$ while for $5 \mathrm{~mm} / \mathrm{s}$ test speed, band propagation velocity ranges about $100 \mathrm{~mm} / \mathrm{s}$. As the tensile test goes on and the plastic strain reaches higher values, the band propagation velocity decreases: the first bands propagate with higher velocity than the last bands, before specimen breaks. Galvanized specimens show band propagation velocities different from non galvanized specimens at the same test speed. For example for $0.05 \mathrm{~mm} / \mathrm{s}$ test speed, band propagation velocity ranges about $10 \mathrm{~mm} / \mathrm{s}$. For these specimens couples of "A" bands nucleate in the center of the specimen, and the two bands propagates towards the opposite ends of the specimens contemporaneously.

Load cell measurements show that lower load values (and lower hardening values) are related to higher band propagation velocities, as shown in figure 7 .

The analysis of thermal data gave band propagation velocities which can be compared to above mentioned optical strain measurement results. In figure 8 the comparison of the data for a a specimen tested at $0,05 \mathrm{~mm} / \mathrm{s}$ is reported as an example.

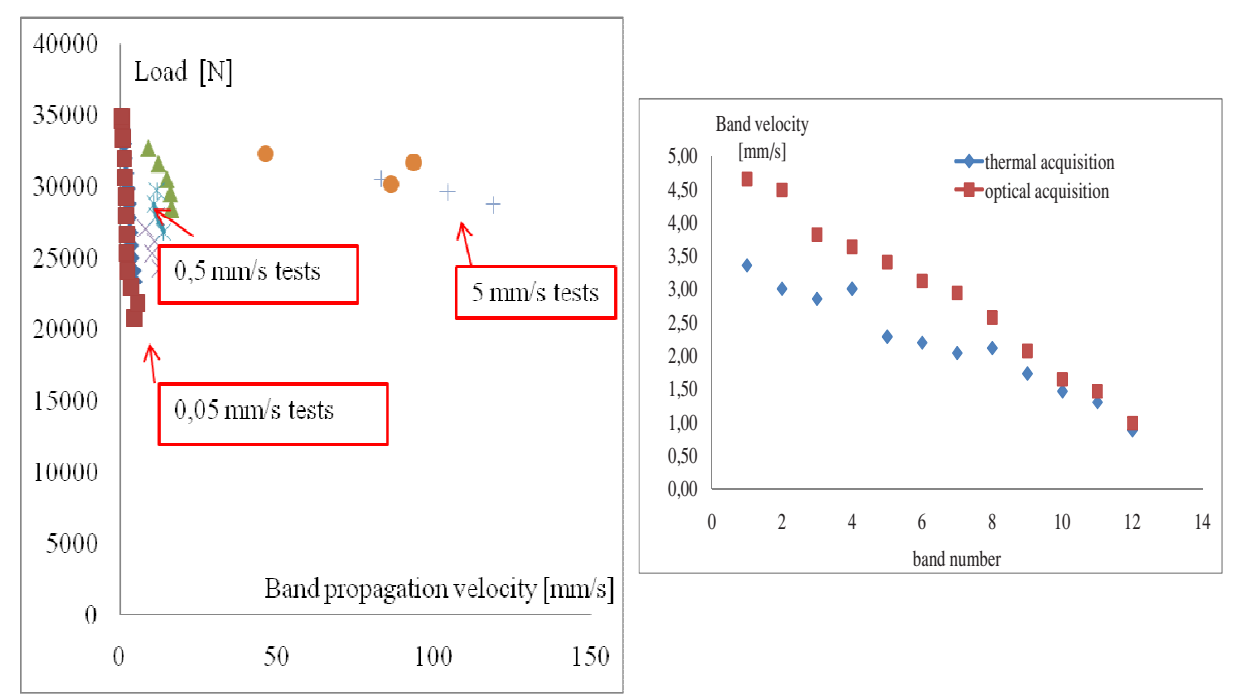

Fig. 7. Load measurements versus band propagation velocity
Fig. 8. Comparison of band propagation velocity results $(0.05 \mathrm{~mm} / \mathrm{s}$ test speed $)$

Both thermography and optical strain measurement results show a decrease in band propagation velocity from the first to the last band. The values of the band propagation velocity calculated by means of thermography are different from optical strain measurements. The difference is higher for higher band propagation velocities, that is for the firstly nucleated bands, and then the difference decreases for the last nucleated bands. This behavior is due to the fact that measurement obtained by means of thermography include the drag effect due to crosshead motion. Measurements by means of optical strain measurements are free from this effect.

In Figure 9 the value of the thermal increment related to each band for all the specimens is shown.

It can be noted that higher test speed causes higher thermal increments. For example for test run at $5 \mathrm{~mm} / \mathrm{s}$ the temperature reaches a value of $95^{\circ} \mathrm{C}$. It can also be noted that for a set test speed, the slower (last nucleated) bands are related to higher temperature increments.

In Figure 10 the number of "A" bands recognized by means of the three methods (optical strain measurement, thermography and load measurement) are compared. The first two methods well discriminate all the bands and the optical system appears to be more accurate with respect to thermography. 


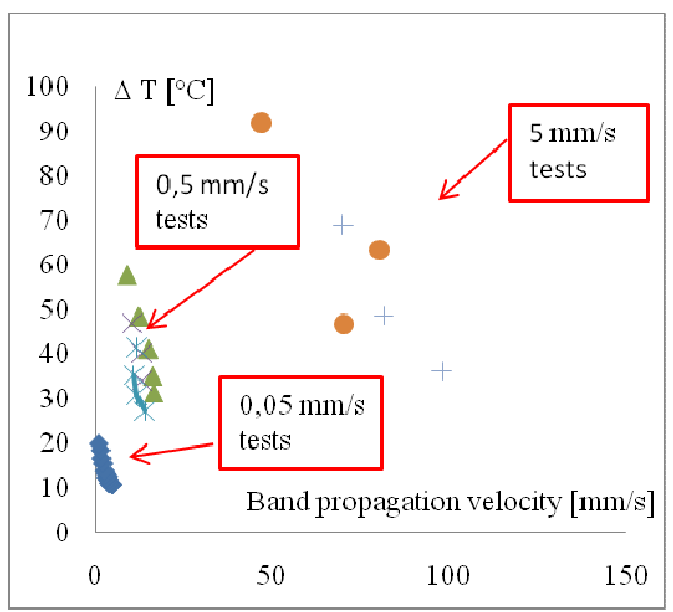

Fig. 9. Thermal increment related to band propagation

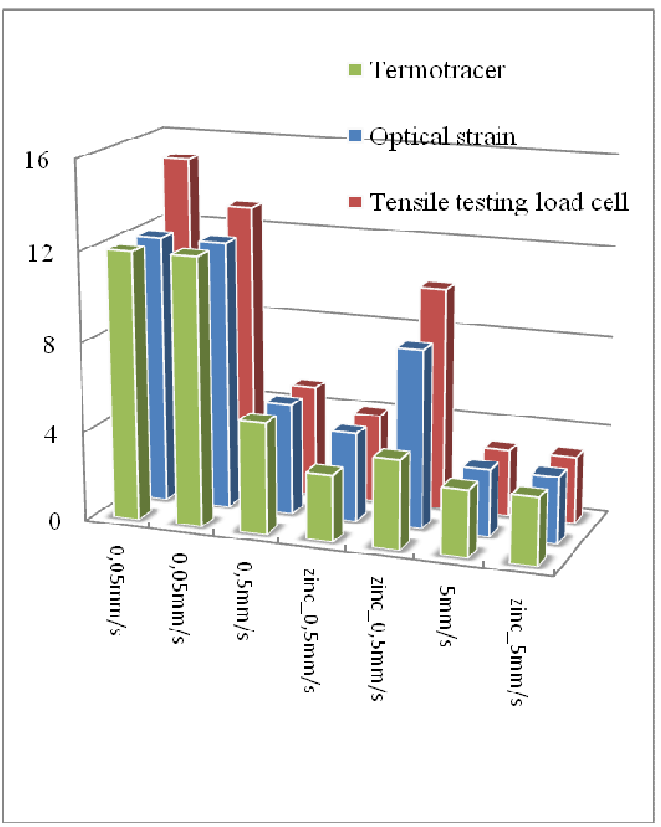

Fig. 10. Comparison of band identification by means of the described methods

\section{Conclusion}

The experimental results presented in the previous section allow to state some points about systems used to identify PLC bands propagating on TWIP steel specimens undergoing tensile testing and about mechanisms of deformation related to PLC effect.

For that concerns microstructural aspects, as the test goes on (and thus plastic deformation), the band propagation velocity decreases. The thermal increment increasing with band nucleation shows that further deformation (that is further band nucleation) causes an increment in dissipation related to subsequent bands. The load increment necessary for further deformation also shows that a higher energy is necessary to further deform the material. This confirms that the grain micro germination and cluster nucleation inside grains, caused by plastic deformation, behave as an obstacle to deformation increment.

The band propagation speed can thus be assumed as an index of the hardening of the material.

Experimental tests allow to confirm that the test speed influences PLC "A" band nucleation; they are numerous for lower test speed and less for higher test speed.

Galvanization too influences both band nucleation mechanism and band propagation velocity.

For that concerns the effectiveness of the two non contact full field band investigation systems, optical strain measurement and thermography, they appear to be complementary. None of them allows to completely describe the phenomenon.

When properly applied, especially for that concerns the data acquisition frequency and procedures, both methods have discriminated all "A" bands at higher test speed and almost all "A" bands for lower test speed.

Both of them have shown that plastic deformation in a TWIP steel specimen undergoing tensile test is concentrated in the PLC band.

Further studies will be dedicated to investigate the strain distribution around the PLC band.

Thermography results are not to be suited for the quantitative measurement of band propagation velocity, while the optical measurement system results to be proper for it. 
This last system results to be suited to measure the value of the strains related to the band while, it is not suited to evaluate energy dissipation related to a band. The measurement of the energy related to a band propagation, that is to an increment of plastic deformation, is possible thanks to thermography.

These two aspects are complementary and useful for further investigation about mechanisms of deformation in TWIP steels.

\section{References}

1. E. Rizzi, P. Haner F., On the Portevin-Le Chatelier effect: theoretical modeling and numerical results, International Journal of Plasticity, 20, 121-165 (2004)

2. D. Cornette, P. Cugy, A. Hildebrand, M. Bouzekri, G. Lovato, Ultra High Strength FeMn TWIP Steels for automotive safety parts, La Revue de Métallurgie, 12, 905-918 (2005)

3. S. Allain, J. P. Chateau, D. Dahmoun, O. Bouaziz, Modeling of mechanical twinning in a high manganese content austenitic steel, Material Science and Enginnering A, 387-389, 272-276 (2004)

4. J. Friedel, Dislocations (Pergamon Press, Oxford, UK, 1964)

5. C. Di Salvo, D. Firrao, P. Matteis, G.Scavino, R. Sesana, Strain and thermal phenomena caused by Portevin Le Chatelier effect in an austenitic steel (in Italian), XXXVIII Convegno AIAS, Italy (2009)

6. H. Louche, A. Chrysocoos, Thermal and dissipative effects accompanying Lüders band propagation, Material Science and Engineering A, 307, 15-22 (2001)

7. A. Chrysochoos, H. Louche, An infrared image processing to analyse the calorific effects accompaniyng strain localisation, International Journal of Engineering Science, 38 (16), 1759$1788(2000)$

8. Lei Chen, Han-Soo Kim, Sung-Kyu Kim, B. C. De Cooman, Localized Deformation due to Portevin-Le Chatelier Effect in 18Mn-0.6C TWIP Austenitic Steel, ISIJ International, 47 (12), 1804-1812 (2007)

9. UNI EN 10002-1: 2004 Metallic materials - tensile test - Part 1: Test method at room temperature 